\title{
ERRATUM: “LYMAN $\alpha$ RADIATIVE TRANSFER IN COSMOLOGICAL SIMULATIONS USING ADAPTIVE MESH REFINEMENT” (2009, ApJ, 696, 853)
}

\section{Peter Laursen ${ }^{1}$, Alexei O. Razoumov ${ }^{2}$, and Jesper Sommer-Larsen ${ }^{1,3}$}

${ }^{1}$ Dark Cosmology Centre, Niels Bohr Institute, University of Copenhagen, Juliane Maries Vej 30, DK-2100 Copenhagen Ø, Denmark; pela@dark-cosmology.dk

${ }^{2}$ Institute for Computational Astrophysics, Department of Astronomy \& Physics, Saint Mary's University, Halifax, NS B3H3C3, Canada; razoumov@ap.smu.ca ${ }^{3}$ Excellence Cluster Universe, Technische Universität München, Boltzmannstraße 2, D-85748 Garching, Germany; jslarsen@astro.ku.dk

In the original paper, we provide a handy functional form for calculating the optimal value of $x_{\text {crit }}$, characterizing the size of the Gaussian core that can be skipped in the acceleration scheme of the radiative transfer, as a function of $a \tau_{0}$ (Equation (17)). Two different sets of parameters are used for $a \tau_{0} \leqslant 60$ and $a \tau_{0}>60$, but in the manuscript, the inequality symbols " $\leqslant$ " and " $>$ " have been misplaced by " $\geqslant$ " and " $<$," respectively. Thus, the correct sentence is:

"Indeed, it is found that the value

$$
x_{\text {crit }}= \begin{cases}0 & \text { for } a \tau_{0} \leqslant 1 \\ 0.02 e^{\xi \ln ^{x} a \tau_{0}} & \text { for } a \tau_{0}>1,\end{cases}
$$

where $(\xi, \chi)=(0.6,1.2)$ or $(1.4,0.6)$ for $a \tau_{0} \leqslant 60$ or $a \tau_{0}>60$, respectively, can be used without affecting the emergent spectrum ..."

The error is only present in the text, and hence no results are affected.

The authors are grateful to Joshua Adams for pointing out the error. 